\title{
More light on membrane protein mobility
}

\section{from Michael T. Flanagan}

ONE of the most useful and unambiguous techniques for measuring the mobility of proteins in a membrane is fluorescence recovery after photobleaching (FRAP). In this technique, a cell is labelled as specifically as possible by saturating a membrane-localised receptor with its ligand which has previously been labelled with a fluorescent dye. As described in the original papers, a small circular spot ( $\sim 8$ $\mu \mathrm{m}$ in diameter) is bleached on the surface of the cell by exposing it to a focused laser beam of the appropriate wavelength to be absorbed by and destroy the fluorescent dye. The appearance of fluorescence within the circle after bleaching can arise only from the diffusion of unbleached material into the spot. The rate of this recovery is related in a relatively simple manner to the diffusion constant of the labelled molecular complex (Edidin et al. Science 191, 466; 1976; Schlessinger et al. Proc. natn. Acad. Sci. U.S.A. 73, 2409; 1976). The essentials of the method were first described by Poo and Cone (Nature 247,438 ; 1974) who, in the absence of laser technology, measured the diffusion constant of rhodopsin in amphibian disk membranes using the easily bleached natural chromophore, retinal. They found a value approximately one order of magnitude less than that found, by resonance techniques, to characterise the lipids of natural membranes. The difference between protein and lipid mobility has emerged as the most interesting theme linking the various FRAP experiments.

A difference might be expected simply because the proteins are much larger than the lipids. However, the first FRAP experiments carried out on mitogens and antibodies bound to cell membrane receptors, for example, concanavalin $A$ on myoblasts, IgE on mast cells, demonstrated that the mobilities of these protein receptor complexes were determined by more than their size. In general two populations of complex were found; one immobile and one with a diffusion constant up to two orders of magnitude lower than that of the membrane lipid. The diffusion constant of the latter population fell dramatically in the presence of cytochalasin B. This microbial metabolite does not alter the mobility of the membrane lipids. As it is known to combine with elements of the cytoskeleton these FRAP results strengthened the growing belief that there is a physical link between some membrane receptors and the cytoskeletal structure underlying the membrane. FRAP has also been used to study lipid mobility in its own right and such studies have given additional

Michael T. Flanagan is with the Biophysics Laboratory at the Standard Telecommunication Laboratories, Harlow, Essox. confidence in the validity of the technique. The diffusion constants obtained using small fluorescent lipid probes confirmed the values obtained from magnetic resonance studies (Wu et al. Biochemistry $16,3939 ; 1977)$. This study also illustrated the limitations of the technique. Diffusion constants in liquid crystalline phases of pure lipids, for example, in dimyristoylphosphatidylcholine above $23^{\circ} \mathrm{C}$, could be measured but not those in the gel phase, that lipid below $23{ }^{\circ} \mathrm{C}$. To extend the detection limit of the technique to cover these high viscosity membranes Smith and McConnell (Proc. natn. Acad. Sci. U.S.A. 75,$2759 ; 1979$ ) introduced a very elegant modification to the technique that has, in addition, assumed considerable importance in examining possible interactions between receptors and cytoskeleton.

Instead of bleaching a spot Smith and McConnell bleached a series of parallel lines by first passing the laser beam through a ruled grating. A square wave pattern is produced which rapidly forms a sine wave pattern. The diffusion constant is obtained from the decay constant of the amplitude of the sine wave. Not only is the sensitivity improved but gross cellular movements can be compensated for as the frequency of the discrete pattern is not altered by proteinreceptor complex or labelled lipid diffusion. McConnell's group have now examined the mobility of the M13 phage coat protein incorporated into an artificial membrane at temperatures both above and below the phase transition temperature of the lipid. The results in the gel phase were compatible with either protein aggregation or phase separation into protein-rich and protein-poor domains. In the liquidcrystalline phase a diffusion constant for the protein comparable with that of the lipid was found (Biochemistry 18, 2256; 1979).

In their next modification to the FRAP method McConnell's group returned to the problem of protein mobility in natural membranes. They have shown that it is possible to measure the anisotropy of diffusion in a membrane by bleaching a two dimensional periodic grid pattern (Proc. natn. Acad. Sci. U.S.A. 76, 5641; 1979). The reduction of the data is no longer simple and requires Fourier image analysis. By aligning one axis of the bleached grid pattern with the predominant direction of the 'stress' fibres of mouse embryo fibroblasts showing anisotropic arrangements in their cytoskeletal components they were able to obtain the diffusion constants of the succinylated concanavalin $\mathrm{A}$ receptor complex in the directions both parallel and perpendicular to the 'stress' fibres. These differed by up to a factor of 10 ; the parallel motion being the faster. The presence of a physical link between this complex and the cytoskeleton now seems certain. Whether it is a direct anchoring link or a more subtle one involving exclusion of the complex from certain regions of the membrane cannot be determined by this technique alone but FRAP clearly will be a powerful tool in the study of the fate of such complexes.

The factors that determine protein mobility in a complex membrane, even in the absence of cytoskeletal interactions, are not fully understood. This is in part because the structure and dynamics of the mixed lipid membranes themselves are not totally clear. But it is also impossible to predict with certainty how a protein will behave in a hypothetical continuous homogeneous two-dimensional fluid. The equations describing diffusion in three dimensions possess neat analytical solutions; when constrained to two dimensions they do not.

Saffman and Delbrück (Proc. natn. Acad. Sci. U.S.A. 72, 3111; 1975) have examined the consequences of this paradox in the context of protein diffusion in a biological membrane. The availability of methods for the accurate determination of both lateral and rotational diffusion constants (correlation spectroscopy for the latter) should now allow the theoretical model derived by these authors to be tested and several of the groups using the FRAP technique see this as one of their goals. Experiments are also being devised to examine the effect of the multicomponent nature of the lipid membrane on protein diffusion. Vaz et al. (Proc. natn. Acad. Sci. U.S.A. 76, 5645; 1979) have taken the apolipoprotein, ApoC-III, as a model of a membrane protein and examined the effect of adding cholesterol to phosphatidylcholine bilayers on the mobility of the protein when bound to such bilayers. They believe that the protein is bound in the head group region of the membrane and that its mobility represents the upper bound for those values that will be found to characterise the integral membrane proteins.

Given the advanced state of the art of membrane reconstitution FRAP studies of several integral proteins are now feasible. In one of the first of these Schindler, Osborn and Koppel (Nature 283, 346; 1980) measured the diffusion constants of the phospholipid, lipopolysaccharide and matrix protein in a reconstituted Escherichia coli outer membrane. They cannot reconcile their results with the model of Saffman and Delbrück and suggest that macromolecular diffusion in a membrane is more analogous to nonelectrolyte diffusion through a polymer network than through a homogeneous fluid. FRAP experiments carried out on reconstituted systems containing enzymes whose activity has been shown to depend on the state or nature of the surrounding lipid, for example the ATP-dependent $\mathrm{Ca}^{2+}$-transporting protein of the sarcoplasmic reticulum, will be of especial interest. 